\title{
Metformin, Effects Beyond Glycemic Control
}

\author{
Soebagijo Adi and Askandar Tjokroprawiro \\ Department of Internal Medicine, Dr. Soetomo Teaching Hospital, Faculty of Medicine, Universitas Airlangga, Surabaya, \\ Indonesia
}

Keywords: Cardiometabolic, Endothelium, Hyperglycemia, Kidney, Metformin, Proinflammatory.

\begin{abstract}
Metformin is an appropriate first-line medication, not only for glycemic control, but also in other situations. Most recently, the spectrum of metformin's target site has expanded to include the endothelium and ovaries. Even if many of these actions are individually modest, they seem to be collectively sufficient to confer therapeutic benefits, not only in cardiometabolism, but also in reproductive aspects related to insulin resistance and proinflammatory states, with promising antitumor and cancer protection properties. The aim of this study was to explain Metformin, Effects Beyond Glycemic Control. In 50 years of its clinical use, there have been no major risks reported with metformin, and serious adverse events attributed to metformin appear to be very low due to some contraindications. The limited use in chronic kidney disease needs to be redefined since many data suggest that metformin may protect against the deleterious consequences of hyperglycemia in the kidneys.
\end{abstract}

\section{INTRODUCTION}

The number of diabetes mellitus (DM) patients in Indonesia and some parts of the world has been increasing (IDF, 2013). Zimmet in XXXX, the president of the International Diabetes Institute, warned that there will be an alarming surge in the number of diabetes patients, from 246 million in 2007 to 380 million by 2025. Zimmet calls this condition "the biggest global epidemic in human history." Diabetes mellitus also closely correlates with high mortality and morbidity due to macro- and micro-vascular complications. The risk of cardiovascular complications reaches 3-fold in DM patients, and life expectancy declines at all ages in line with the disease severity. Mortality risk doubles for DM patients aged in their $40 \mathrm{~s}$ to $50 \mathrm{~s}$. Microvascular complications are the major cause of blindness, renal failure and amputations that all require expensive treatment (Turner et al., 1996).

Blood sugar level control plays a very important role in preventing DM complications. The high incidence of complications in DM patients shows inadequate therapy (Turner et al., 1996). In developed countries like the United States, only 34\% of DM patients can achieve the target of $\mathrm{HbA}_{1 \mathrm{c}}<7 \%$ (Grant et al., 2005). The 1999-2000 National Health Examination Survey (NHANES) found only $7 \%$ of adult $\mathrm{DM}$ patients achieved $\mathrm{HbA}_{1 \mathrm{c}}<7 \%$, blood pressure $<130 / 80 \mathrm{mmHg}$ and total cholesterol $<200$ $\mathrm{mg} / \mathrm{dl}$ (Saydah et al., 2004).

A steno-2 study reported that blood glucose control, multifactor interventions (hypertension and dyslipidemia) gave better results in preventing and reducing cardiovascular risk and mortality (Gaede. P. et al., 2008). This aim of this study was to explain Metformin, Effects Beyond Glycemic Control.

\section{CARDIOVASCULAR COMPLICATION EPIDEMIOLOGY IN TYPE-2 DIABETES MELLITUS}

Cardiovascular disease (CVD), coronary artery disease (CAD), cerebrovascular disease and peripheral vascular disease (PVD) are the major macrovascular complications in diabetes mellitus type 2 (DMT2) that are responsible for more than $50 \%$ of mortality cases in diabetes patients (Singer et al., 1992).

The relative risk (RR) of mortality from CAD is estimated to increase 1.5-2.5-fold in male DM patients and more than four times in female DM patients compared with non-DM patients of the same age. It seems that the natural protection women have for CVD disappears in diabetes (Nathan et al., 
1997). Macrovascular complications are not specific to DMT2 as many other risk factors also play a role even before DM clinical diagnosis is established. Diabetes patients have a CVD risk 2-4-fold higher (Ramachadran and Snehalatha, 2007).

In the United States, diabetes is the 4th most common cause of death, and coronary heart disease (CHD) accounts for nearly $75 \%$ of all mortalities in DM patients. The United Kingdom Prospective Diabetes Study (UKPDS) reported that fatal CVD was 70-fold more frequent than microvascular complications after follow up for 9 years. Diabetes mellitus patients do not only carry a greater risk for CHD, but also the fact that CHD will appear at a younger age than the non-DM population. In addition, DM patients also have a high risk of multivessel disease with decreased coronary flow reserve. Cardiovascular events in DM are also followed by a poor prognosis. ATP III considers diabetes as equivalent to $\mathrm{CHD}$ based on studies reporting high cardiovascular risk in diabetes patients (Ramachadran and Snehalatha, 2007).

The Asia Pacific Cohort study shows that increased fasting blood glucose will be followed by increased risk for stroke, ischemic heart disease and cardiovascular death. Microvascular complications are more typical for diabetes. Two important risk factors for microvascular complications are blood sugar and blood pressure level. Microangiopathy disturbs the function of several organs, including blindness from retinopathy, terminal renal failure from nephropathy, cardiomyopathy leading to heart failure, and dementia caused by microangiopathy in the brain (Marre et al., 2007).

In the U.S., diabetic nephropathy accounts for $40 \%$ of terminal renal failure cases. Approximately $20-30 \%$ of patients with type- 1 or type- 2 DM show progression to nephropathy (American Diabetes Association, 2003).

Retinopathy is the leading cause of blindness. Its prevalence is largely determined by diabetes duration and the control of blood glucose and blood pressure. Diabetes duration is the strongest predictor of retinopathy. In the Wisconsin Epidemiologic Study of Diabetic Retinopathy (WESDR), the prevalence of retinopathy was $8 \%$ at 3 years, before rising to $25 \%$ at 5 years, $60 \%$ at 10 years and $80 \%$ at 15 years (Fong et al., 2003).

\section{BLOOD SUGAR LEVEL AND CARDIOVASCULAR COMPLICATION CONTROLS: EVIDENCE FROM EPIDEMIOLOGY STUDIES}

The correlation between cardiovascular complications and elevated blood sugar level has been proved by numerous studies. In the last three decades, experimental studies on animals, observational studies in humans and clinical trials have been conducted to determine the correlation between hyperglycemia and diabetes complication onset.

Diabetes Control and Complications Trial (DCCT) and other studies with similar designs and smaller samples of the Stockholm Diabetes Intervention Study have reported that decreased blood glucose level can delay and inhibit microvascular complication progression in type-1 DM, with risk reduction varying from $35-75 \%$ (American Diabetes Association, 2003).

In DCCT, blood sugar level improvement is also associated with decreased cardiovascular events, but this decrease is not significant. In type-2 DM, the UKPDS is the largest and longest study to date, involving 5,102 newly diagnosed type-2 DM patients in 23 UK medical centers between 1977 1991.

The UKPDS found that intensive therapy with $7 \% \mathrm{HbA}_{1 \mathrm{c}}$ could reduce overall microvascular complications by $25 \%$. These data showed that hyperglycemia plays a very important role in microvascular complications. Further analysis showed that each $1 \%$ decrease in $\mathrm{HbA}_{1 \mathrm{c}}$ was associated with a reduced risk of microvascular complication by $35 \%$.

For macrovascular complication risk, UKPDS showed that decreased blood glucose level could reduce macrovascular complication risk by $16 \%$, but this decrease was not significant. However, the epidemiological analysis of UKPDS suggested an association between cardiovascular complications and blood glucose level. Every $1 \%$ decrease in $\mathrm{HbA}_{1 \mathrm{c}}$ is followed by a $25 \%$ decrease in diabetesrelated deaths, $7 \%$ reduction in all-cause mortality, and an $18 \%$ reduction in both fatal and non-fatal myocardial infarcts (American Diabetes Association, 2003).

The Kumamoto study compared multiple insulin injection therapy ( 3 or more daily insulin injections) with conventional insulin injection therapy (one or two daily insulin intermediate-acting injections) in 
type-2 DM patients and found that retinopathy risk decreased by $47 \%$ for every $1 \%$ decrease in $\mathrm{HbA}_{1 \mathrm{c}}$. Albuminuria risk decreased by $74 \%$ for every $2 \%$ decrease in $\mathrm{HbA}_{1 \mathrm{C}}$ (Shichiri et al., 2000).

The Kumamoto study argued that the threshold limit value for preventing microvascular complication onset and progression was $\mathrm{HbA}_{1 \mathrm{c}}$ $<6.5 \%$, fasting blood glucose $<110 \mathrm{mg} / \mathrm{dl}$, and 2 hour post-prandial glucose $<180 \mathrm{mg} / \mathrm{dl}$ (Shichiri et al., 2000).

The Asia Pacific Cohort Studies Collaboration reported that each fasting glucose decrease of 1 $\mathrm{mmol} / \mathrm{l}$ was associated with decreased stroke risk by $21 \%$ and decreased ischemic heart disease by $23 \%$ in Asia-Pacific populations. This decrease is similar between Asian populations and Australia and New Zealand (ASIA PACIFIC COHORT, 2004).

The EPIC-Norfolk Study involved non-diabetic populations and examined the effects of $\mathrm{HbA}_{1 \mathrm{c}}$ on CHD, CVD and death risks. The study used 4,662 male and 5,570 female subjects, with a follow up of 6 years. This study showed increased the relative risks of CHD in men and women were 1.25 and 1.20 , respectively, CVD in men and women were 1.21 and 1.21 , respectively and total mortality in men and women were 1.24 and 1.28 , respectively, for each $1 \% \mathrm{HbA}_{1 \mathrm{c}}$ increase above 5\% (adjusted for age and other risk factors) in a non-diabetic population without any history of CVD, diabetes, or $\mathrm{HbA}_{1 \mathrm{c}} \geq 7 \%$ (Kay-Tee Khaw et al., 2004). presented in Figure 1 (American Diabetes Association, 2016).

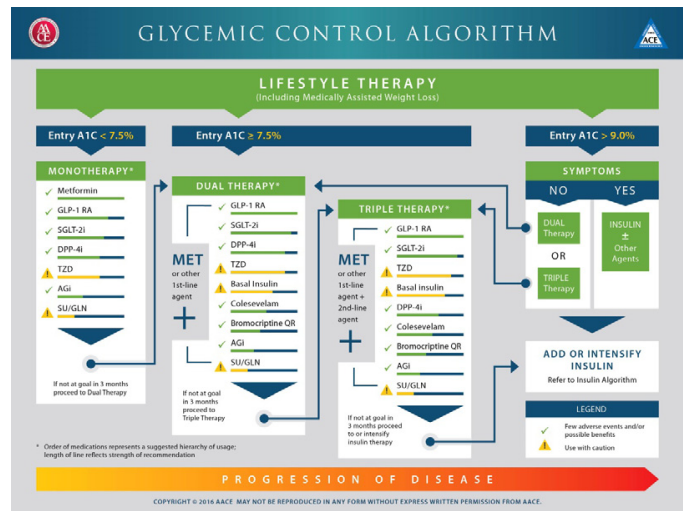

Figure 1: Management algorithm from the American Association of Clinical Endocrinology (AACE) and American College of Endocrinology (ACE) 2016 (AACE/ACE 2016).

The ADA algorithm still applied metformin as the foundation of therapy from 2006 to 2016. Metformin became the first-line therapy. On the next line, there is always a combination of metformin therapy, unless there is a contra indication of metformin use. The role of metformin as the foundation of DMT2 therapy is also listed in the algorithm of the 2016 American Association of Clinical Endocrinology, suggesting that metformin occupies an important position in DMT2 treatment.

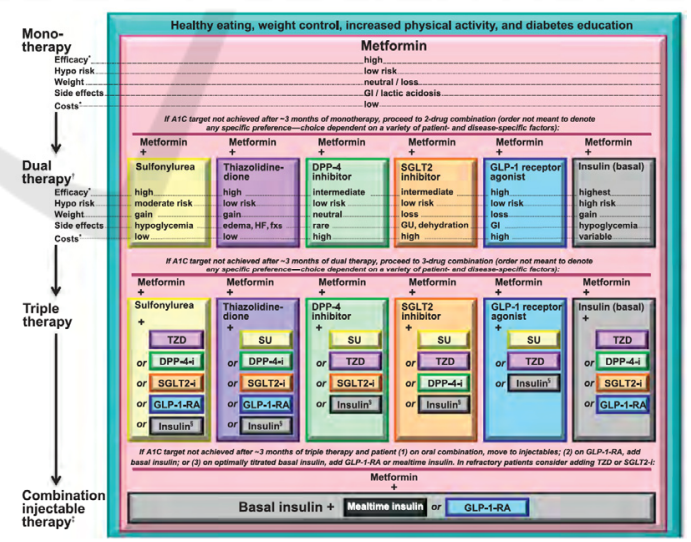

Figure 2: Medical management of hyperglycemia in DMT2 (American Diabetes Association, 2016).

Metformin was discovered more than 50 years ago by Jean Sterne, a professor working at Laennec Hospital, Paris. Metformin's reputation grew slowly. Metformin was not popularly predicted as a major cure in DM management from 1960-1980, even though many newer medicines were in development. 
In 2007, metformin became an option along with lifestyle modification.

Metformin has become less favorable due to its phenformin side-effects. Disavantages of metformin include, lactic acidosis side-effects from biguanide. However, at the end of 1960-1970, Scots reported metformin effectiveness as a single or combination therapy with sulfonylurea, but only in 1995, the FDA declared that metformin was relatively safe. The UKPDS also noted that metformin had beneficial effects of metabolic and cardiovascular improvement.

Metformin, included in the biguanide group (Figure 1), has efficacy in preventing endothelial tissue damage in hyperglycemic states. This efficacy is not only obtained due to pharmacologically antihyperglycemic properties, but also an inhibitory effect in regard to endothelial cell damage (Manaf et al., 2010; Viollet B, 2012; Sing, 2011).

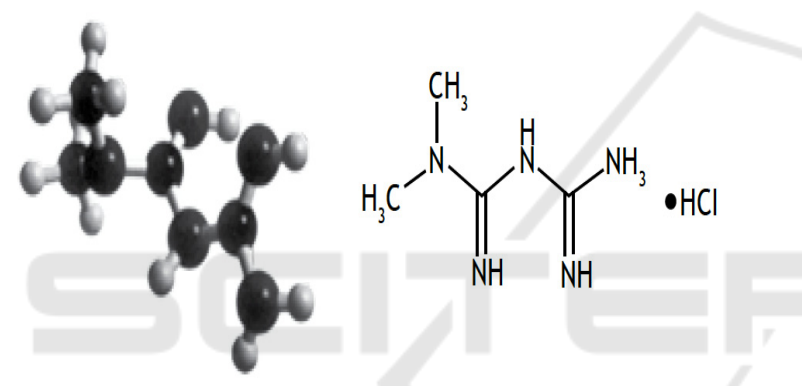

Figure 3: Biochemical structure of metformin: 1,1dimethylbiguanide hydrochloride.

\section{METFORMIN WORKING MECHANISM}

The main mechanism of metformin in DMT2 patients is the decrease of liver glucose production by lowering gluconeogenesis, but also the increase of glucose uptake by smooth muscle (Sing, 2011)

Metformin activates AMPK (adenosine monophosphate protein kinase) in liver and muscles; therefore, it improves glucose and lipid metabolism in DMT2. AMPK decreases SREBP-1 (sterol regulatory element-binding protein 1) expression that serves as a transcription factor involving insulin resistance pathogenesis, dyslipidemia and diabetes. AMPK activation is an important step in decreasing metformin-mediated liver glucose production.

Recent studies have shown that AMPK upstream regulators are protein kinases known as LKB1 (liver kinase B1). LKB1 has been known to be a tumor suppressor. Metformin has an effect on the liver and muscle, thereby lowering fasting blood glucose level and postprandial glucose (Sing, 2011).

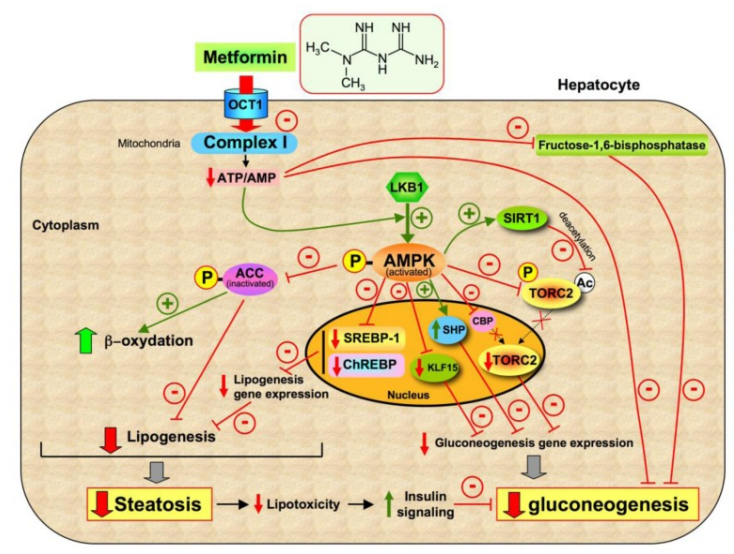

Figure 4: Metformin mechanism (Viollet B, 2012) OCT1: Organic Cation Transporter 1; LKB1: Liver Kinase B1; SIRT1: Sirtuin1; AMPK: Adenosine Monophosphate Protein Kinase; ACC: acetyl CoA carboxylase; TORC2: transducer of regulated CREB protein 2; SEREBP-1: Sterol Regulatory Element-Binding Protein 1; ChREBP: Carbohydrate-responsive element-binding protein; CBP: CREB Binding Protein; KLF15: Krüppel-like factor 15.

Some of the important effects of metformin include: 1) metformin acts as an antihyperglycemic by improving insulin sensitivity in the liver and a few effects in peripheral tissue resulting from several mechanisms, such as increased insulin receptor tyrosine kinase (IRTK) activity, increased GLUT-4 amount and activity, enhanced glycogen synthesis; 2) metformin decreases liver glucose production by inhibiting gluconeogenesis and possible glycogenesis; 3) metformin increases glucose uptake, glycogen storage and glucose oxidation by insulin; 4) metformin prevents lipolysis; 5) metformin protects pancreatic islets from injury caused by high glucose (glucotoxicity) and/or free fatty acids (lipotoxicity); 6) patients treated with metformin DMT2 exhibit decreased PAI-1, decreased triglycerides plasma, LDL and slightly increased HDL; 7) metformin demonstrates fibrinolytic and anti-thrombotic effects, including decreased fibrinogen, decreased PAI-1, lowered FVII, and lowered F-XIIIa.

\section{METFORMIN EFFECTS ON ADIPONECTIN}

Adamia reported that 6-month metformin therapy in postmenopausal DMT2 women significantly 
increased adiponectin levels along with decreased BMI. Hypoadiponectinemia in postmenopausal women is caused by insulin resistance. Adiponectin levels increase along with improvements in insulin action due to metformin for 6 months (Kirpichnikov and McFarlane, 2002).

Increased adiponectin level due to metformin was also found in a study conducted by Manaf. Administration of metformin $2 \times 500 \mathrm{mg}$ for 12 weeks significantly raised adiponectin levels compared to the control group. Decreases in adiponectin level in the control group were believed to be due to increased insulin resistance. The metformin mechanism in increasing adiponectin levels is suspected to be through adenosine enzyme activation.

AMPK administration also decreases TNF $\alpha$ and interleukin-6 levels, before indirectly increasing adiponectin production and secretion. Therefore, both mechanisms will improve insulin resistance and inhibit prediabetic conversion into DMT2 (Manaf et al., 2010).

\section{METFORMIN EFFECTS ON VASCULAR PROTECTION}

Diabetes closely correlates to increased cardiovascular risk. In addition to lowering blood glucose, diabetes treatment should consider drugs that may also reduce cardiovascular risk and cardiovascular heart disease.

There has been abundant evidence pointing out that not only one cellular mechanism plays a role in the metformin cardioprotective effect. Some evidence suggests that metformin has a beneficial effect on microvasculature and provides macrovascular protection.

Metformin improves survival by reducing mortality risk from all causes, myocardial infarction risk, stroke, re-infarction, angina symptoms, acute coronary syndrome and decreasing infarct size (Tjokroprawiro, 2009).

\section{METFORMIN ROLES IN OBESITY}

Metformin is the first-line treatment in overweight and obese diabetics. Metformin significantly decreases cardiovascular risk in obese DM patients (Sing, 2011). Some studies have shown that metformin provides significant weight loss. The anorectic effects of metformin play a role in weight loss.

\section{ANTIHYPERTENSION EFFECTS OF METFORMIN}

Several reports have found decreased blood pressure during metformin therapy, both systolic and diastolic. The decreased blood pressure is presumably via central sympathoinhibitory action (Sing, 2011). Metformin therapy significantly improves sympathovagal cardiac balance, but does not affect mean arterial blood pressure (MABP). Long-term studies are necessary to reveal metformin effects on decreased blood pressure.

\section{METFORMIN EFFECTS ON LIPID ACCUMULATION IN METABOLIC SYNDROME}

Metformin demonstrates decreased lipid accumulation in macrophages by suppressing FOXO1-mediated FABP4 transcription. Therefore, metformin has a protective effect for lipid accumulation in macrophages and is a treatment option with the additional effect of preventing and improving atherosclerosis in metabolic syndrome.

Metformin also increases carnitine palmitoyltransferase-1 (CPT-1) expression and decreases fatty acid-binding protein 4 (FABP4) expression that plays a role in palmitate (PA)induced lipid accumulation at the transcriptional level (Sing, 2011).

\section{METFORMIN REDUCES ANDROGEN LEVELS IN PCOS}

Metformin has shown the effect of lowering hyperinsulinemia and hyperandrogenemia in patients with polycystic ovarian syndrome (PCOS). Metformin also improves the insulin response against oral glucose tolerance. Metformin plays a direct role in thecal cell decrease as it decreases steroid production. Several studies have reported that metformin has a direct inhibitory effect on androstenedione production in human ovarian thecal-like androgen-producing tumor cells (Sing, 2011). 


\section{METFORMIN REDUCES OXIDATIVE STRESS AND PLATELET ACTIVATION}

Oxidative stress and increased platelet activation play a role in atherosclerosis acceleration in DM patients. A random study by Formosa et al. showed that 12-week metformin therapy in DMT2 patients significantly reduced 2 alpha and 11-dehydrothromboxane B excretion in urine, and significantly increased vitamin A and E levels (Sing, 2011).

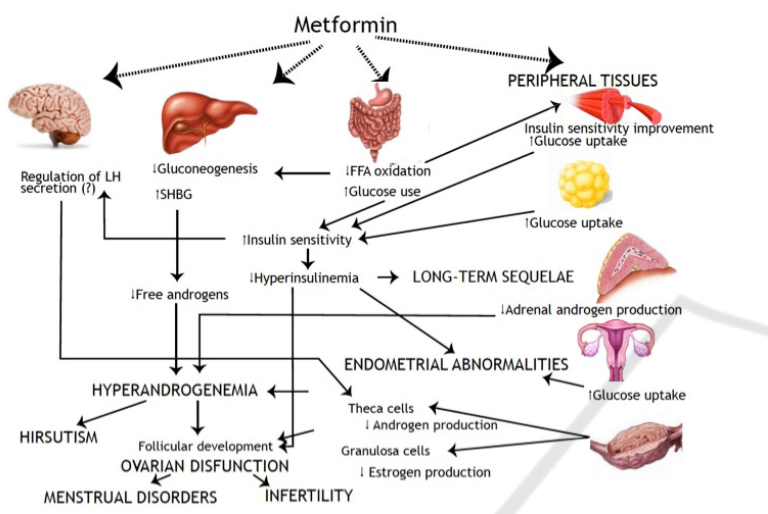

Figure 5: Metformin mechanism (Sing, 2011).

\section{ANTITUMOR EFFECTS OF METFORMIN}

Metformin activates AMPK through two different pathways, oxidative resistance/electron phosphorylation transport that subsequently results in a decreased ATP/AMP ratio and/or through direct LKB1 activation path (Figure 2). As an additional effect of the protein synthesis inhibitory effect through mTOR resistance, AMPK activation increases the CD8 T lymphocytes' memory and suppresses cancer cachexia signal in the hypothalamus. Electron transport resistance can result in cancer cell death (Figure 4).

Metformin has been shown to increase the CD8 $\mathrm{T}$ cell memory, and can improve anti-cancer vaccine effectiveness.

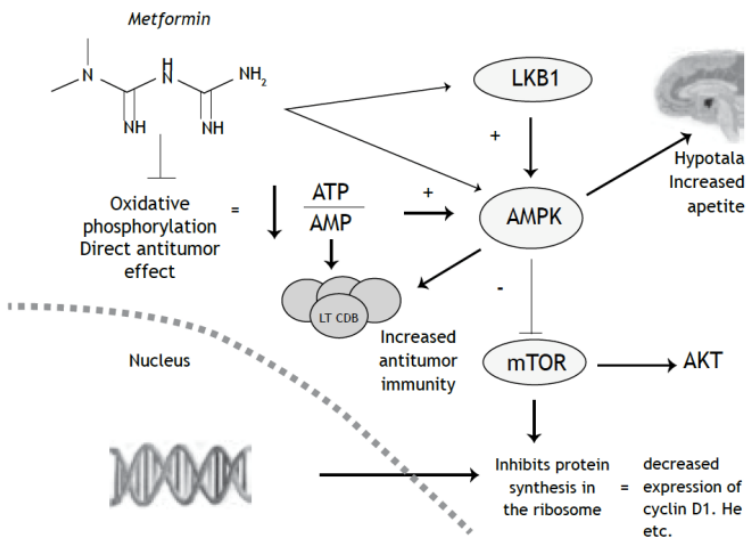

Figure 6: Metformin antitumor mechanism.

\section{METFORMIN AND DIABETIC NEPHROPATHY}

Around $20-40 \%$ of diabetic patients will experience diabetic nephropathy. Since metformin is excreted through the kidneys, the decreased clearance of metformin is a lactic acidosis risk. Dose adjustment is recommended if the estimated glomerular filtration rate $<45 \mathrm{ml} / \mathrm{min} / 1.73 \mathrm{~m}^{2}$, and metformin administration is stopped when estimated glomerular filtration rate $<45 \mathrm{ml} / \mathrm{min} / 1.73 \mathrm{~m}^{2}$. Even though metformin clearance decreases in CKD, the metformin level in blood remains within the therapeutic range at eFGR $>30 \mathrm{ml} / \mathrm{min} / 1.73 \mathrm{~m}^{2}$ and does not significantly affect blood lactate level (Sing, 2011).

However, the latest data show that metformin has a renoprotective effect on hyperglycemia in the kidneys. Takiyama et al., in their study on rats showed that metformin $250 \mathrm{mg} / \mathrm{kg} /$ day for 9-39 weeks prevented tubular injury from hyperglycemia, while insulin showed no renoprotection (Viollet B, 2012).

In this study, metformin specifically decreased HIF-1 expression, not only through decreased ATP synthesis, but also reduced oxygen consumption from renal cells. This effect persisted despite AMPK $\alpha$ subunit knockdowns. In this condition, AICAR or rapamycin (mTORC1 inhibitor) could not provide a similar effect as metformin.

Metformin even can prevent possible gentamycin-induced renal failure through decreased ROS-mediated lipid peroxidation, and decreased TGF $\beta$-induced epithelial-to-mesenchymal transition (EMT), which plays a role in tubulointerstitial fibrosis in diabetic nephropathy. 


\section{REFERENCES}

AACE/ACE 2016. comprehensive Type 2 Diabetes Management Algorithm 2016. . Endocr Pract., ;22:, 84-113.

AMERICAN DIABETES ASSOCIATION 2016; Standard of medical care in diabetes - 2016. . Diabetes Care. , 39, S1-S112

AMERICAN DIABETES ASSOCIATION, P. S. 2003. Implications of the United Kingdom Prospective Diabetes Study. . Diabetes Care, 26 S28-S32.

AMERICAN DIABETES ASSOCIATION, P. S. 2003; . Diabetic Nephropathy. . Diabetes Care 26, S94-S98.

ASIA PACIFIC COHORT, S. C. 2004;. Blood Glucose and Risk of Cardiovascular Disease in the Asia Pacific Region. . Diabetes Care 27:, 2836-2842.

FONG, D. S., AIELlO, L., . \& GARDNER, T. W., . ET.AL. 2003; Diabetic Retinopathy. ADA position statement. . Diabetes Care. , 26 2836-2842.

GAEDE. P., LUND-ANDERSEN, H. \& PARVING, H., AND PEDERSEN O. 2008; . Effect of a Multifactorial Intervention on Mortality in Type 2 Diabetes. . $N$ Engl J Med 358:, 580-91.

GRANT, R. W., BUSE, J. B., . \& MEIGS, J. B. 2005;. Quality of Diabetes Care in U.S.Academic Medical Centers. . Diabetes Care 28:, 337-342.

KAY-TEE KHAW., WAREHAM, N., BINGHAM, S., LUBEN, R., WELCH , A., , \& DAY, N. 2004. Association of Hemoglobin A1c with Cardiovascular Disease and Mortality in Adults: The European Prospective Investigation into Cancer in Norfolk. Ann Intern Med., 141:, 413-420.

KIRPICHNIKOV, D., . \& MCFARLANE, S. I. S., J.. 2002;. Metformin : an update. . Annals of Internal Medicine 137:, 25-33

MANAF, A., DESWITA, S. \& SYAHBUDDIN, S. 2010. Pengaruh metformin terhadap kadar adiponektin pada pasien prediabetes dengan obesitas. Surabaya Metabolic Syndrome Update-6 (SUMETSU-6) Metabolic Cardiovascular Disease Surabaya Update6 (MECARSU-6). . Surabaya, .

MARRE, M., TRAVERT, F. \& BEKHERRAZ, A. 2007;. ADVANCE: the biggest ongoing randomized controlled trial of cardiovascular disease in type 2 diabetes. An interview. Medicographia, 29:, 207-212.

NATHAN, D. M., MEIGS, J. \& SINGER, D. E. 1997; The epidemilogy of cardiovascular disease in type 2 diabetes mellitus: how sweet it is ..or is it? Lancet 350, 4-9.

RAMACHADRAN, A. \& SNEHALATHA, C. 2007. Epidemiology of heart disease in type 2 diabetes. Medicographia. , 29:, 207-212.

SAYDAH, S., FRAKIN, J. \& COWIE, C. C. 2004. Poor Control of Risk Factors for Vascular Disease Among Adults With Previously Diagnosed Diabetes. . JAMA 291:, 335-342.

SHICHIRI, M., KISHIKAWA, H., OHKUBO, Y. \& WAKE, N. 2000. Long-Term Results of the Kumamoto Study on Optimal Diabetes Control in
Type 2 Diabetic Patients. . Diabetes Care, 23, B21B29.

SING, J. 2011. Metformin Beyond Glycemic. Medicine Update-2011.

SINGER, D. E., NATHAN, D. M. \& ANDERSON, K. M., ET AL. 1992;. Association of HbA1C with prevalent cardiovascular disease in the originalcohort of the Framingham Heart Study. Diabetes Care, 41:, 202208.

TJOKROPRAWIRO, A. 2009. . A memorial review article for the 15-year clinical experiences with metformin (It is more than an oral anti diabetes agent). - Surabaya Metabolic Syndrome Update-5 (SUMETSU-5) Metabolic Cardiovascular Disease Surabaya Update-4 (MECARSU-4) Surabaya, 8-10 Mei.

TURNER, R., CULL, C. \& R., H. 1996 United Kingdom Prospective Diabetes Study 17: A 9-year Update of Randomized, Controlled Trial on the Effect of Improved Metabolic Control on Complications in Non-Insulin-dependent Diabetes Mellitus. . Annals of Internal medicine 124:, 136-145.

VIOLLET B, G. B., GARCIA NS, LECREC J, FORETTZ M, ANDREELLI F. 2012, . Cellular and molecular mechanisms of metformin: an overview. ,. Clin Sci (Lond). , 122, 253-270. 